Pacific Journal of Mathematics

CONCERNING PERIODIC SUBADDITIVE FUNCTIONS 


\title{
CONCERNING PERIODIC SUBADDITIVE FUNCTIONS
}

\author{
R. F. JOLLY
}

The author investigates those subsets $M$ of the complex plane with the group property that $M$ is closed with respect to complex multiplication. In particular if $M$ is closed, bounded and has for its boundary a curve given in polar form by $\rho(\theta)=r(\theta) \exp (i \theta)$ where $r$ is a positive continuous function with period $2 \pi$, then $r$ is characterized by these requirements, together with the additional condition that $r$ be submultiplicative. If $f(x)=-\log r(x)$, the corresponding conditions on $f$ are: $f$ is a continuous nonnegative subadditive function with period $2 \pi$.

Some relations between the roots (zeros) and periods of subadditive functions are discussed and in particular, it is shown that: if $f$ is a continuous subadditive function not identically zero, with period 1 and with a root $c$ (i.e., $f(c)=$ 0 ), then $c$ is a rational number $m / n$ (in lowest terms), $f(0)=$ 0 and $f$ has period $1 / n$.

For each positive number $c$ and function $f$ on the set of all numbers, a type of polygonal approximation $P(c, f)$ is defined such that if $f$ is continuous, $\lim P(c, f)=f$ uniformly over every bounded number set as $c \rightarrow 0$. If $f$ is subadditive, $P(c, f)$ is subadditive. The subadditive $P(c, f)$ are characterized in terms of their slopes. Since a change of scale does not affect the subadditive property, the author studies functions with period 1 rather than those with period $2 \pi$. For each positive integer $n$, the collection $K_{n}$ of all functions $P(1 / n, f)$ for all continuous subadditive functions $f$ with period 1 , is shown to have a finite basis. In fact, $K_{n}$ forms a function cone with finitely many extremal elements (the basis). While an explicit representation is not given, the proof shows how these extremal elements may be constructed.

Several examples are given to illustrate some pathological cases. The methods of this paper may easily be applied to the solution of certain other functional inequalities with corresponding restrictions.

1. Introduction. The statement that $M$ is a $G$-set means that $M$ is a point set in the complex plane such that if $P$ is in $M$ and $Q$ is in $M$, then the product $P Q$ is in $M$, i.e., $M$ has the group property that it is

Received March 5, 1964. Presented to the American Mathematical Society on August 29, 1963. Part of this work is contained in the author's dissertation at the University of Texas. The author is deeply grateful to Professor H.S. Wall for his suggestions about the preparation of this manuscript. 
closed with respect to multiplication. For example, the set of all points ${ }^{1}$ $z$ such that $|z|<1$ is a $G$-set; for each number $k$, the intersection of the interiors of the circles with radius $\left(1+k^{2}\right)^{1 / 2}$ and centers $i k$ and $-i k$ is a $G$-set; and the union of the coordinate axes is a $G$-set which contains no domain $(\S 6)$. The statement that $M$ is a simple $G$-set means that $M$ is a $G$-set which is closed, bounded and which has for its boundary a curve given in polar form by $\rho(\theta)=r(\theta) \exp (i \theta)$ where $r$ is a positive continuous function of period $2 \pi$. The function $r$ is completely characterized by these requirements together with the additional condition that for each number $x$ and number $y, r(x) r(y) \leqq$ $r(x+y)$. If $f(x)=-\log r(x)$, the corresponding conditions on $f$ are: $f$ is continuous, nonnegative and of period $2 \pi$ and moreover, for each number $x$ and number $y, f(x+y) \leqq f(x)+f(y)$. Hence, the determination of all simple $G$-sets resolves itself into the problem of the determination of all continuous nonnegative subadditive functions with period $2 \pi(\S 2)$.

For each number $p$, let $F_{p}$ denote the collection of all functions $f$ which are subadditive on the set of all numbers and which have the property that if $x$ is a number, $f(x+p)=f(x)$. Let $F$ denote the collection of all continuous functions in $F_{1}$. The statement that $c$ is a root of $f$ means $f(c)=0$. An anchored function is one with zero for a root. In $\S 3$, some relationships are shown between the roots and periods of subadditive functions. In particular, it is shown that if $f$ is a function in $F$ not identically zero and $c$ is a root of $f$, then $c$ is a rational number $m / n$ (in lowest terms) and $f$ is an anchored function with period $1 / n$ (Theorem 5 ).

Since a change of scale does not affect the subadditive property (Lemma 3), we study the functions in $F_{1}$ instead of those in $F_{2 \pi}$ and thereby simplify the notation. For each positive number $c$ and function $f$ on the set of all numbers, a type of polygonal approximation $P(c, f)$ is defined $(\S 4)$ such that if $f$ is continuous, $\lim _{c \rightarrow 0} P(c, f)=f$ uniformly over every bounded number set. These polygonal approximations to functions in $F_{0}$ are themselves in $F_{0}$ (Theorem 6) and are characterized in terms of their slopes (Theorem 7). It is then shown that for each positive integer $n$, the collection $K_{n}$ of all functions $P(1 / n, f)$ for all functions $f$ in $F$, has a finite basis in the sense that there is an integer $M(n)$ and a sequence $\alpha_{n}$ of $M(n)$ elements of $K_{n}$. such that a function $g$ belongs to $K_{n}$ if, and only if, $g$ is the sum of a linear combination of the functions of $\alpha_{n}$ with nonnegative coefficients (Theorem 9). These polygonal subadditive functions are then used to characterize $F$ as the collection to which $f$ belongs if, and only if, $f$

1 In this paper, the word number shall be used to denote a real number and the word point shall mean a point of the complex plane. 
can be approximated uniformly by linear combinations of the functions of $\alpha_{1}, \alpha_{2}, \alpha_{3}, \cdots$ with nonnegative coefficients.

There are simple $G$-sets which are not convex and in fact, which have no tangent at any point (Theorem 12). Some other examples, which show the difficulty in obtaining nontrivial characterizations of $G$-sets, are given in $\S 6$. One example is a countable $G$-set dense in the plane.

2. Boundaries of simple $G$-sets. Throughout this section, assume that $r$ is a positive continuous function with period $2 \pi$ and let $D$ denote the closed and bounded set with boundary $z=r(\theta) \exp (i \theta)$.

THEOREM 1. The following two statements are equivalent:

(i) $D$ is a simple G-set.

(ii) For each number $\alpha$ and each number $\beta, r(\alpha) r(\beta) \leqq r(\alpha+\beta)$.

Proof. To show that statement (ii) implies statement (i), let $P=$ $c \exp (i \alpha)$ and $Q=d \exp (i \beta)$ where $0 \leqq c<r(\alpha), 0 \leqq d<r(\beta)$ and $r(\alpha) r(\beta) \leqq r(\alpha+\beta)$. Hence $P Q=c d \exp (i(\alpha+\beta))$ and $P Q$ is in $D$.

To show that statement (i) implies statement (ii), assume (i) and $r(\alpha) r(\beta)>r(\alpha+\beta)$. Let $P=(r(\alpha)-\delta) \exp (i \alpha)$ and $Q=(r(\beta)-\delta)$ $\exp (i \beta)$ where $\delta=[r(\alpha) r(\beta)-r(\alpha+\beta)] /[r(\alpha)+r(\beta)]>0$. Note that $r(\alpha)-\delta=\left[r^{2}(\alpha)+r(\alpha+\beta)\right] /[r(\alpha)+r(\beta)]>0$. Therefore $P$ is in $D$. Likewise $r(\beta)-\delta=\left[r^{2}(\beta)+r(\alpha+\beta)\right] /[r(\alpha)+r(\beta)]$ and $Q$ is in $D$. Since $P$ is in $D$ and $Q$ is in $D . \quad P Q$ is in $D$. Therefore $r(\alpha+\beta)>$ $(r(\alpha)-\delta)(r(\beta)-\delta)$ but $(r(\alpha)-\delta)(r(\beta)-\delta)>r(\alpha) r(\beta)-\delta(r(\alpha)+$ $r(\beta))=r(\alpha+\beta)$. This is a contradiction.

THEOREM 2. Suppose that $D$ is a G-set. Then the following two statements are equivalent:

(i) Each point of $D$ is the product of two points of $D$.

(ii) $r(0)=1$.

Proof. $r$ is continuous and has period $2 \pi$, therefore there is a number $0 \leqq w<2 \pi$ such that for any number $\alpha, r(\alpha) \leqq r(w)$. Since $r^{2}(w) \leqq r(2 w) \leqq r(w), r(w) \leqq 1$.

To show that statement (ii) implies statement (i), suppose $Z=d$ $\exp (i \alpha)$ where $0 \leqq \mathrm{~d}<r(\alpha)$. From the preceding $d<r(\alpha) \leqq r(w) \leqq$ 1 , hence $d<(1 / 2)(1+d)<1$ and $2 d /(1+d)<1$. Let $W=(1 / 2)(1+d)$ $\exp (i \alpha)$ and $U=2 d /(1+d)$. Then $Z=W U$.

To show that statement (i) implies statement (ii), assume (i) is true. Let us first show that $r(w)=1$. Suppose $r(w)<1$. Let $Z=$ $(1 / 2)\left[r(w)+r^{2}(w)\right] \exp (i w)$. By (i) there is a point $W=c \exp (i \alpha)$ and a point $U=d \exp (i \beta)$ where $0 \leqq c<r(\alpha), 0 \leqq d<r(\beta)$ and $Z=$ 
$W U$. Note that $0 \leqq c<r(\alpha) \leqq r(w)$ and $0 \leqq d<r(\beta) \leqq r(w)$. Therefore $c d<r^{2}(w)<(1 / 2)\left[r(w)+r^{2}(w)\right]<r(w)$. Hence $Z \neq W U$ which is a contradiction. Therefore $r(w)=1$.

Since $1=r(w) r(k w) \leqq r((k+1) w) \leqq r(w)=1$ when $r(k w)=1$, it follows by induction that if $n$ is a positive integer, $r(n w)=1$.

Since $r$ is continuous and periodic, $r$ is uniformly continuous. Suppose $\varepsilon>0$. Then there is a number $\delta>0$ such that if $|x-y|<$ $\delta,|r(x)-r(y)|<\varepsilon$. For infinitely many positive integers $m$ and $n$, $|(w / 2 \pi)-(m / n)|<n^{-2}<\delta /(2 \pi n)$. For such integers $m$ and $n, \mid r(n w)-$ $r(2 \pi m) \mid<\varepsilon, r(n w)=1$ (previously proven) and $r(2 \pi m)=r(0) \quad(r$ has period $2 \pi)$. Hence, $1-r(0)<\varepsilon$. Therefore $r(0)=1$.

THEOREM 3. If $g$ is a continuous periodic submultiplicative function, either $g$ is a positive function or $g \equiv 0$.

Proof. For each number $x, 0 \leqq g(x / 2) g(x / 2) \leqq g(x)$. Since $g$ is continuous, either $g \equiv 0$ or there is some segment containing no root of $g$. Suppose $a<b$ and the segment $(a, b)$ contains no root of $g$. If $a<x<b$ and $n>0$, then $n a<n x<n b$ and if $g(k x) \neq 0$ and $g(x) \neq$ 0 , then $0<g(x) g(k x) \leqq g((k+1) x)$. It follows by induction that for every positive integer $n$, the segment $(n a, n b)$ contains no root of $g$. Since $g$ has period $p$, for some positive number $p$, and there is a positive integer $N$ such that $N(b-a)>p$, there is no root of $g$ and hence $g$ is positive.

THEOREM 4. Suppose $f$ and $g$ are functions such that $f=-\log$ g. Then the following two statements are equivalent:

(i) $g$ is positive and submultiplicative.

(ii) $f$ is subadditive.

The proof is omitted.

3. Roots and periods of subadditive functions. Let us now show how the roots of subadditive functions are related to their periods and in particular, what happens in the continuous case.

Note that if $p$ and $-p$ are both roots of the function $f$ of $F_{0}$, then $f$ is an anchored function with period $p$. This is shown by the inequalities

$$
\begin{aligned}
0=f(p) & \leqq f(p)+f(0)=f(0)=f(p-p) \leqq f(p)+f(-p)=0 \\
& f(x)=f(x+p-p) \leqq f(x+p)+f(-p) \leqq f(x)+f(p)=f(x) .
\end{aligned}
$$

On the other hand, the example

$$
f(x)=\{1, \text { if } x \leqq \pi / 2 ;|\sin x|, \text { if } \pi / 2 \leqq x\}
$$


shows that a continuous element of $F_{0}$ may have infinitely many roots without being either anchored or periodic. However, if $f$ is a nonnegative continuous element of $F_{0}$ with both a positive and negative root, then $f$ is anchored and periodic. This is easily shown by letting $p$ and $q$ denote respectively the smallest positive and largest negative roots of $f$ (these obviously exist). But $q<p+q<p$ and $0 \leqq f(p+$ $q) \leqq f(p)+f(q)=0$. Hence $f(p+q)=0$ and $p+q=0$.

THEOREM 5. Suppose $f$ is in $F$ and $c$ is a number different from 0 such that $f(c)=0$. Then

(i) $f$ is anchored;

(ii) if $c$ is irrational, $f \equiv 0$;

(iii) if $c$ is a rational number $m / n$ (in lowest terms), then $f$ has period $1 / n$.

Proof. First we show that there is no number $x$ such that $f(x)<0$. Suppose there were such an $x$. Then $f(0+0) \leqq f(0)+f(0)$ implies $0 \leqq f(0)$. Hence $x \neq 0$. Since $f((k+1) x) \leqq f(x)+f(k x)$, it follows by induction that $f(n x) \leqq n f(x)$ for every positive integer $n$. As $f$ is continuous and periodic, there is a number $M$ such that if $w$ is a number, $|f(w)|<M$. Let $m$ denote an integer greater than $M /|f(x)|$. Therefore $m|f(x)|>M$ but if $f(x)<0,|f(m x)|=-f(m x) \geqq-$ $m f(x)=m|f(x)|$ which is a contradiction.

Since $f$ has period 1 , assume $0<c<1$. It was previously shown that $f$ is nonnegative and if $n$ is a positive integer, $f(n x) \leqq n f(x)$. Hence $f(n c)=0$ for every positive integer $n$. Following the line of argument used in Theorem 2, the fact that $f$ is uniformly continuous may now be used to show that $f(0)=0$ and therefore $f$ is anchored.

To show (ii), assume $c$ is irrational and $f \neq 0$. Again following a line of argument used in Theorem 2 , the fact that the multiples of $c$ modulo 1 are dense in the interval $[0,1]$ gives a contradiction since $f$ is continuous, has period 1 and $f(n c)=0$ for every positive integer $n$.

To show (iii), assume $c$ is the rational number $m / n$ in lowest positive terms. There exists an integer $k$ and an integer $p$ such that $\mathrm{km}-n p=1$. Hence $\mathrm{km} / n=(1+n p)=p+1 / n$ and $0=f(\mathrm{~km} / n)=$ $f(p+1 / n)=f(1 / n)$. For each number $w, f(w+1 / n) \leqq f(w)+f(1 /$ $n)=f(w)$. Therefore it follows by induction that for every number $x$ and positive integer $s, f(x+s / n) \leqq f(x+1 / n) \leqq f(x)$. But if $s=$ $n, f(x)=f(x+n / n) \leqq f(x+1 / n) \leqq f(x)$. Therefore $f$ has period $1 / n$.

4. Certain polygonal approximations to subadditive functions. Let us start with some elementary properties of subadditive functions and follow this with a definition and some properties of a certain type 
of polygonal function.

Lemma 1. If $a>0, b>0, f$ is in $F_{p}$ and $g$ is in $F_{p}$, then $a f+b g$ is in $F_{p}$.

LeMma 2. If for each positive integer $n, f_{n}$ is in $F_{p}$ and $g$ is a function such that for each number $x, f_{n}(x)$ converges to $g(x)$, then $g$ is in $F_{p}$.

Lemma 3. If $c$ is a number, $f$ is in $F_{c p}$ and $g$ is the function such that for every number $x, g(x)=f(c x)$, then $g$ is in $F_{p}$.

Proofs to Lemmas 1, 2 and 3 may be found in [3, Chap. VII].

Definition. For each positive number $c$ and function $f$ defined on the set of all numbers, let $P(c, f)$ denote the function $h$ such that (i) if $n$ is a positive integer and $n c-c \leqq x \leqq n c$, then $h(x)=m_{n}(x-$ $n c)+f(n c)$ where $m_{n}=[f(n c)-f(n c-c)] / c$ and (ii) if $k$ is a negative integer and $k c \leqq x \leqq k c+c$, then $h(x)=-m_{k}(x-k c)+f(k c)$ where $m_{k}=-[f(k c+c)-f(k c)] / c$. Also let $m_{0}=f(0) / c$.

Definition. For each function $h$ defined on the set of all numbers, let $h^{*}$ denote the transformation from the set of all ordered number pairs into a number set such that for every ordered number pair ( $x$, $y), h^{*}(x, y)=h(x)+h(y)-h(x+y)$.

TheOREM 6. If $f$ is in $F_{0}$ and $c$ is a number then $P(c, f)$ is in $F_{0}$.

This theorem is equivalent to Theorem 8 of [2].

Notation. When $n$ is negative, let

$$
\sum_{p=0}^{n} m_{p}=\sum_{p=n}^{0} m_{p}=\sum_{p=0}^{|n|} m_{-p} .
$$

THEOREM 7. If $f$ is a function defined on the set of all numbers. and $c$ is a positive number, then $P(c, f)$ is in $F_{0}$ if, and only if, for every integer $n$ and integer $k$,

$$
\sum_{p=0}^{n+k} m_{p} \leqq \sum_{p=0}^{n} m_{p}+\sum_{p=0}^{k} m_{p} .
$$

Proof. For each positive integer $n, m_{n}=[f(n c)-f(n c-c)] / c$. Hence $c m_{n}=f(n c)-f(n c-c)$ and $f(n c)=c m_{n}+f(n c-c)$. It follows by induction that 


$$
f(n c)=c \sum_{p=1}^{n} m_{p}+f(0)=c \sum_{p=0}^{n} m_{p} .
$$

For each negative integer $k, m_{k}=-[f(k c+c)-f(k c)] / c$. Hence $c m_{k}=-f(k c+c)+f(k c)$ and $f(k c)=c m_{k}+f(k c+c)$. It follows by induction that

$$
f(k c)=c \sum_{p=k}^{-1} m_{p}+f(0)=c \sum_{p=0}^{k} m_{p} .
$$

In the proof of Theorem 6 , it is shown that $P(c, f)$ is in $F_{0}$ if, and only if, for every integer $n$ and integer $k, f(n c)+f(k c)-f((n+$ $k) c) \geqq 0$. Hence the theorem follows.

This theorem can be used to derive several of the well-known theorems concerning the rate of growth of subadditive functions. Notice that one could easily restrict the domain of $f$ to the positive or negative numbers. Note also the obvious fact that if $f$ has period $n c$, then $\sum_{p=1}^{n} m_{p}=0$.

THEOREM 8. If $f$ is a function defined on the set of all numbers and $\left\{c_{n}\right\}$ is a number sequence converging to 0 such that $\left\{P\left(c_{n}, f\right)\right\}$ converges pointwise to $f$, then $f$ is in $F_{0}$ if , and only if, $P\left(c_{n}, f\right)$ is in $F_{0}$ for every positive integer $n$.

Proof. From Theorem 6, it follows that if $f$ is in $F_{0}$ and $n$ is a positive integer, then $P\left(c_{n}, f\right)$ is in $\mathrm{F}_{0}$.

Under the hypothesis of the theorem, if $P\left(c_{n}, f\right)$ is in $F_{0}$ for every positive integer $n$, then by Lemma $2, f$ is in $F_{0}$.

5. Periodic polygonal subadditive functions. A type of polygonal approximation to elements of $F$ is described and these are shown to have a finite basis. In fact, these polygonal approximations (for a fixed $n$ ) form a function cone with finitely many extremal elements (the basis). While an explicit representation is not given, the proof of Theorem 9 shows how these extremal elements may be constructed.

THeORem 9. For each positive integer $n$, there is an integer $M(n)$ and a finite sequence $\left\{\alpha_{n p}\right\}$ with $M(n)$ terms such that

(i) if $p$ is an integer and $1 \leqq p \leqq M(n)$, then for some function $f$ in $F, \alpha_{n p}=P(1 / n, f)$ and

(ii) if $g$ is in $F$ and $h$ is the function $P(1 / n, g)$, then there is a sequence $\left\{a_{p}\right\}$ of nonnegative numbers such that $h=\sum_{p=1}^{M(n)} a_{p} \alpha_{n p}$.

Proof. Suppose $n$ is a positive integer. Let $F^{\prime}$ denote the collection to which $h$ belongs if, and only if, there is a function $f$ in $F$ 
such that $h=P(1 / n, f)$. Note that if $h$ is in $F^{\prime}, h$ is continuous, $h(1+p / n)=f(1+p / n)=f(p / n)=h(p / n)$ for each integer $p$ and hence, $h$ has period 1. By Lemma 1 , if $a \geqq 0, g$ is in $F^{\prime}$, and $h$ is in $F^{\prime \prime}$, then $a g+h$ is in $F^{\prime}$. It follows by induction that any linear combination with nonnegative coefficients of functions in $F^{\prime}$ is itself in $F^{\prime}$.

Let $Z_{0}$ denote the collection of all points $(p / n, k / n)$ for $p=1,2$, $3, \cdots, n$ and $k=1,2,3, \cdots, n$. For each $h$ in $F^{\prime}, h^{*}(x+1, y)=$ $h^{*}(x, y+1)=h^{*}(x, y)$. Making use of part of the proof of Theorem $6, h^{*}$ is nonnegative if, and only if, $h^{*}$ is nonnegative at every point of $Z_{0}$. Each point $(p / n, k / n)$ of $Z_{0}$ such that $h^{*}(p / n, k / n)=0$, is called a zero of $h^{*}$. If $h$ is in $F^{\prime}, h$ is said to be fundamental only if for each function $g$ in $F^{\prime}$ such that $h-g$ is in $F^{\prime \prime}$, there is a nonnegative number $c$ such that $g=c h$.

Let us now show that when $h$ is in $F^{\prime}$, the statement that $h^{*} \equiv$ 0 is equivalent to the statement that $h \equiv 0$. If $h \equiv 0$, then $h^{*} \equiv 0$. Suppose $h^{*} \equiv 0$. Then for every number $x, h(2 x)=2 h(x)$. Therefore by induction $h(x)=(2 x) / 2=\cdots=h\left(n 2^{n}\right) / 2^{n}$ for every positive integer $n$. Since $h$ is continuous and periodic, there is a number $B$ such that for any number $w,|h(w)|<B$. As $0 \leqq h(x)<B / 2^{n}$ for every positive integer $n, h \equiv 0$.

Next let us show that if $f$ is in $F^{\prime}$ and $g$ is in $F^{\prime}$ then the statement that $f^{*}=g^{*}$ is equivalent to the statement that $f=g$. If $f=g$, then $f^{*}=g^{*}$. If $f^{*}=g^{*}$ and $h=f-g$, then $h^{*}=(f-g)^{*}=$ $f^{*}-g^{*} \equiv 0$ but from the preceding $h \equiv 0$ and hence $f=g$.

Next let us show that the function $h$ in $F^{\prime}$ is fundamental if, and only if, it is true that if $g$ is in $F^{\prime}$ and every zero of $h^{*}$ is a zero of $g^{*}$, then every zero of $g^{*}$ is a zero of $h^{*}$.

Case 1. Suppose $h$ is not fundamental. Then there is a function $g$ in $F$ such that $h-g$ is in $F^{\prime}$; yet there is no nonnegative number $c$ such that $g=c h$. Note that there is no zero $z$ of $h^{*}$ which is not a zero of $g^{*}$ as $(h-g)^{*}$ would be negative at $z$, which is impossible by a previous result. There is a least upper bound $c$ of all numbers $d$ such that $h-d g$ is in $F^{\prime}$. By Lemma $2, h-c g$ is in $F^{\prime}, h-c g \not \equiv$ 0 by assumption. If $h^{*}-c g^{*}$ is positive at every point $z$ of $Z_{0}$ which is not a zero of $h^{*}$, then there is a number $d>c$ such that $h^{*}-d g^{*}$ is positive at every such point $z$, which would contradict the fact that $c$ is the largest number such that $h-c g$ is in $F^{\prime}$. Hence $h-c g$ is a function such that every zero of $h^{*}$ is a zero of $(h-c g)^{*}$ but some zero of $(h-c g)^{*}$ is not a zero of $h^{*}$. Note that since $Z_{0}$ is finite, it now follows by induction that there is some fundamental function $f$ such that every zero of $h^{*}$ is a zero of $f^{*}$. For each $h$ in $F^{\prime}$ such that $h^{*}$ has a zero, let $Z_{h}$ denote the set to which $z$ belongs only if $z$ is a zero of $h^{*}$. 
Case 2. Suppose $g$ is in $F^{\prime \prime}, g \not \equiv 0$ and $Z_{h}$ is a proper subset of $Z_{g}$. There is a positive number $c$ such that the product of $c$ and the maximum value of $g^{*}$ on $Z_{0}$ is less than the smallest positive value of $h^{*}$ on $Z_{0}$. Therefore $h^{*}-c g^{*}$ is nonnegative at each point of $Z_{0}$; consequently $h-c g$ is in $F^{\prime}$. But if $h-d g$ is in $F^{\prime}$ for some number $d$, then, as there is some zero $z$ of $g^{*}$ which is not a zero of $h^{*}$, $h^{*}-d g^{*}$ is positive at $z$ and $h-d g \not \equiv 0$. Therefore $h$ is not foundamental.

Let $C$ denote the collection to which $f$ belongs only if $f$ is a fundamental function such that $\sum_{p=1}^{n} f(p / n)=1$. For each function $h$ in $F$ except 0 , there is a function $f$ in $C$ such that $Z_{h}$ (if it exists) is a subset of $Z_{f}$. If $f$ is a fundamental function and $g$ is a fundamental function such that $Z_{f}=Z_{o}$ then by a previous argument, there is a positive number $c$ such that $f=c g$. Therefore if $f$ and $g$ are fundamental functions in $C, Z_{f} \neq Z_{g}$ and neither is a subset of the other. Since $Z_{0}$ is finite, $C$ is finite. Let $M(n)$ denote the number of functions in $C$ and arrange these functions in a sequence $\left\{\alpha_{n p}\right\}$.

It has been previously shown that any linear combination with nonnegative coefficients of elements of $F$ is itself an element of $F$. Hence there remains only to show that every element $h$ of $F^{\prime \prime}$ can be represented as a linear combination with nonnegative coefficients of the functions $\left\{\alpha_{n p}\right\}$. Let $h_{1}=a_{1} \alpha_{n 1}$ where $a_{1}$ is the largest number $c$ such that $h-c \alpha_{n 1}$ is in $F^{\prime}$. For each positive integer $p \leqq M(n)$, let $h_{p}=h_{p-1}+a_{p} \alpha_{n p}$ where $a_{p}$ is the largest number $c$ such that $h-h_{p-1}-$ $c \alpha_{n p}$ is in $F^{\prime}$.

$$
h_{M(n)}=\sum_{p=1}^{M(n)} a_{p} \alpha_{n p} .
$$

Let

$$
g=h-h_{M(n)}=h-\sum_{p=1}^{M(n)} a_{p} \alpha_{n p}
$$

Unless $g \equiv 0$ there is an integer $k$ such that $Z_{g}$ is a subset of $Z_{\alpha_{n k}}$. There is a largest number $d$ such that $g-d \alpha_{n h}$ is in $F^{\prime} . h_{h}=h_{k-1}+$ $a_{k} \alpha_{n k}$ where $a_{k}$ is the largest number $c$ such that $h-h_{k-1}-c \alpha_{n k}$ is in $F^{\prime}$. But if $g-d \alpha_{n k}$ is in $F^{\prime}$, then $\left(h-h_{n-1}-c \alpha_{n k}\right)-d \alpha_{n k}$ is in $F^{\prime}$ which is a contradiction. Therefore $g \equiv 0$.

THEOREM 10. There is a sequence $\left\{\alpha_{n}\right\}$ of functions in $F$ such that $f$ belongs to $F$ if, and only if, there exists a sequence $\left\{g_{n}\right\}$ converging uniformly to $f$ such that for each positive integer $n$, there is a sequence $\left\{a_{n p}\right\}$ of nonnegative numbers such that $g_{n}=\sum_{p=1}^{n}$ $a_{n p} \alpha_{p}$.

Proof. Let $\left\{\alpha_{n}\right\}$ denote a sequence of functions in $F$ such that 
for each positive integer $n$ and positive integer $p \leqq M(n)$, there is a positive integer $k$ such that $\alpha_{k}=a_{n p}$ where $\alpha_{n p}$ is the $p$ th term of the sequence $\left\{\alpha_{n p}\right\}$ of Theorem 9 .

If $f$ is in $F$, the sequence $\{D(1 / n, f)\}$ converges uniformly to $f$. For each positive integer $k$, there is an integer $n(k)$ and a sequence $\left\{a_{n(k) p}\right\}$ of nonnegative numbers such that $P(1 / k, f)=\sum_{p=1}^{n(k)} a_{n(k) p} \alpha_{p}$ (by Theorem 9). Let $g_{m}=\sum_{p=1}^{n(k)} a_{n(k) p} \alpha_{p}$ if $n(k) \leqq m<n(k+1)$. Then $\left\{g_{m}\right\}$ converges uniformly to $f$.

By using the fact that the sum of two continuous functions with period 1 , is a continuous function with period 1 , Lemma 1 , and induction, it follows that if for some sequence $\left\{a_{p}\right\}$ of nonnegative numbers and some integer $n, g_{n}=\sum_{p=1}^{n} a_{n p} \alpha_{p}$, then $g$ is in $F$. If $\left\{g_{n}\right\}$ converges uniformly to $f$, then $f$ is a continuous function with period 1 and by Lemma $2, f$ is in $F$.

6. Some examples and comments. The examples in Theorem 11 are typical of the fundamental anchored polygonal elements in $F$; the example in Theorem 12 shows that some functions in $F$ are pathological.

THEOREM 11. Suppose $0<k<1$ and $f$ is the function with period 1 such that if $0 \leqq x \leqq k, f(x)=x(1-k)$ and if $k \leqq x \leqq 1$, $f(x)=k(1-x)$. Then $f$ is in $F$.

This theorem can be shown by computing $f^{*}$. It is quite easy to establish that $f^{*} \geqq 0$.

It follows from Theorem 11 and a well-known characterization of continuous convex functions on an interval that if $n$ is a positive integer and $f$ is a nonnegative convex function on $[0,1 / n]$, then $f$ can be extended to be subadditive with period $1 / n$. This result should appear in the Pacific Journal in a paper by Richard Laatsch using different methods (private communication).

THEOREM 12. There exists a totally nondifferentiable function in $F$.

Proof. It follows from Theorem 11 that $y=|\operatorname{Arcsin}(\sin \pi x)|$ represents a function in $F$. In a different setting and using a different notation, there is a proof in $[1, \mathrm{p} .115]$ that the function $f$ defined by $f(x)=\sum_{n=1}^{\infty}\left|\operatorname{Arcsin}\left(\sin 2^{n} \pi x\right)\right|\left(\pi 2^{n}\right)$ is totally nondifferentiable. By Theorem 10, $f$ is in $F$.

Notice that the graph of $Z=f(\theta) \exp (i \theta)$ forms the boundary of a simple $G$-set with no tangent at any point. 
As might be expected, when one considers $G$-sets which are not necessarily simple, one finds some very complicated examples. The following should illustrate this and also, should show some of the aspects of $G$-sets.

Suppose $M$ is a point set and $M^{\prime}$ is the set to which $Z$ belongs if, and only if, for some point $W$ of $M$ and number $0 \leqq t \leqq 1, Z=t W$, i.e., $M^{\prime}$ is the smallest star-shaped set about the origin which covers $M$. A slight modification of the argument for Theorem 1 would show that if $M$ is a $G$-set, then $M^{\prime}$ is a $G$-set. Moreover, a modification of the argument for Theorem 2 would show that if $M$ is a $G$-set, then $M$ is bounded if, and only if, $M$ is a subset of the unit disc, i.e., if $Z$ is in $M,|Z| \leqq 1$.

That the set $M^{\prime}$ need not contain a domain can be seen by taking, for some positive integer $n, M$ to be then $n$th roots of 1 .

Even when $M^{\prime}$ is a bounded $G$-set, there is no requirement that its boundary be the graph of $Z=r(\theta) \exp (i \theta)$ for some positive continuous function with period $2 \pi$. This can be seen by taking $M$ to be, for some number $k \neq 0$, the graph of $Z=\exp ((k+i) \theta)$ for $0 \leqq$ $\theta<2 \pi$.

Suppose $R$ is a number set and $g$ is a function defined on $R$ such that if $x$ is in $R$ and $y$ is in $R$, then $x+y$ is in $R$ and $g(x) g(y)=$ $g(x+y)$. Then if $M$ denotes the set of all points $Z=g(x) \exp (i x)$ for all numbers $x$ in $R, M$ is a $G$-set. It is well-known that such sets $R$, and even that countable sets $R$, exist along with additive functions $f$ defined on $R$ which are dense in the plane. If $g(x)=$ $\exp (f(x))$ then the corresponding set would be dense in the plane.

The methods of this paper may easily be applied to certain other functional inequalities. For example, analogous theorems hold for the solutions to $f(2 x) \leqq 2 f(x)$ and in most cases the arguments do not need to be changed.

\section{REFERENCES}

1. R. P. Boas, A primer of real functions, Mathematical Association of America, Carus Monographs, Vol. 13, 1960.

2. Andrew Bruckner, Minimal superadditive extensions of superadditive functions, Pacific J. Math., 10 (1960), 1155-1162.

3. E. Hille, and R. Phillips, Functional analysis and semi-groups, Amer. Math. Soc., Colloquium Publications, Vol. 31, 1957.

UNIVERSITY OF TEXAS AND

University of CALIForNia, Riverside 



\section{PACIFIC JOURNAL OF MATHEMATICS}

\section{EDITORS}

H. Samelson

Stanford University

Stanford, California

R. M. Blumenthal

University of Washington

Seattle, Washington 98105
J. Dugundu

University of Southern California Los Angeles, California 90007

*Richard Arens

University of California

Los Angeles, California 90024

\section{ASSOCIATE EDITORS}
E. F. BECKENBACH
B. H. NeumanN
F. WOLF
K. YOSIDA

\section{SUPPORTING INSTITUTIONS}

UNIVERSITY OF BRITISH COLUMBIA CALIFORNIA INSTITUTE OF TECHNOLOGY UNIVERSITY OF CALIFORNIA MONTANA STATE UNIVERSITY

UNIVERSITY OF NEVADA

NEW MEXICO STATE UNIVERSITY

OREGON STATE UNIVERSITY

UNIVERSITY OF OREGON

OSAKA UNIVERSITY

UNIVERSITY OF SOUTHERN CALIFORNIA
STANFORD UNIVERSITY

UNIVERSITY OF TOKYO

UNIVERSITY OF UTAH

WASHINGTON STATE UNIVERSITY

UNIVERSITY OF WASHINGTON

AMERICAN MATHEMATICAL SOCIETY CALIFORNIA RESEARCH CORPORATION SPACE TECHNOLOGY LABORATORIES NAVAL ORDNANCE TEST STATION 


\section{Pacific Journal of Mathematics}

\section{Vol. 15, No. $1 \quad$ September, 1965}

Donald Charles Benson, Unimodular solutions of infinite systems of linear

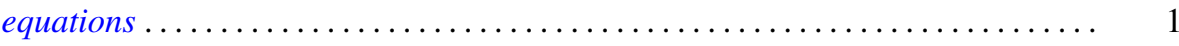

Richard Earl Block, Transitive groups of collineations on certain designs . . . . . . 13

Barry William Boehm, Existence of best rational Tchebycheff approximations .... . 19

Joseph Patrick Brannen, A note on Hausdorff's summation methods . . . . . . . . . . 29

Dennison Robert Brown, Topological semilattices on the two-cell ............ 35

Peter Southcott Bullen, Some inequalities for symmetric means . . . . . . . . . . 47

David Geoffrey Cantor, On arithmetic properties of coefficients of rational

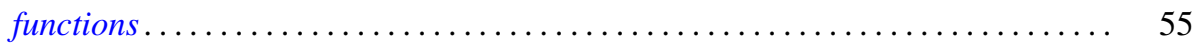

Luther Elic Claborn, Dedekind domains and rings of quotients . . . . . . . . . 59

Allan Clark, Homotopy commutativity and the Moore spectral sequence ........ 65

Allen Devinatz, The asymptotic nature of the solutions of certain linear systems of

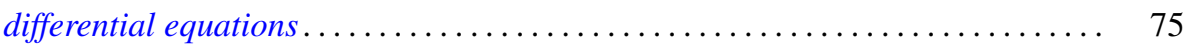

Robert E. Edwards, Approximation by convolutions ................... 85

Theodore William Gamelin, Decomposition theorems for Fredholm operators . . . . . 97

Edmond E. Granirer, On the invariant mean on topological semigroups and on

topological groups .................................. 107

Noel Justin Hicks, Closed vector fields . . . . . . . . . . . . . . . . . . . 141

Charles Ray Hobby and Ronald Pyke, Doubly stochastic operators obtained from

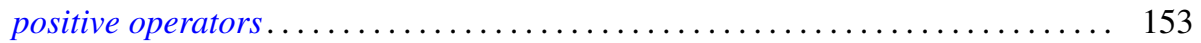

Robert Franklin Jolly, Concerning periodic subadditive functions . . . . . . . . . 159

Tosio Kato, Wave operators and unitary equivalence . . . . . . . . . . . . . . 171

Paul Katz and Ernst Gabor Straus, Infinite sums in algebraic structures . . . . . . . 181

Herbert Frederick Kreimer, Jr., On an extension of the Picard-Vessiot theory ...... 191

Radha Govinda Laha and Eugene Lukacs, On a linear form whose distribution is

identical with that of a monomial ......................... 207

Donald A. Ludwig, Singularities of superpositions of distributions . . . . . . . . . 215

Albert W. Marshall and Ingram Olkin, Norms and inequalities for condition

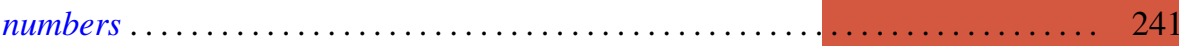

Horace Yomishi Mochizuki, Finitistic global dimension for rings . . . . . . . . . . 249

Robert Harvey Oehmke and Reuben Sandler, The collineation groups of division

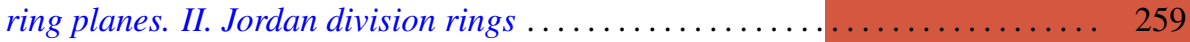

George H. Orland, On non-convex polyhedral surfaces in $E^{3} \ldots \ldots \ldots \ldots \ldots \ldots \ldots 267$

Theodore G. Ostrom, Collineation groups of semi-translation planes . . . . . . . . 273

Arthur Argyle Sagle, On anti-commutative algebras and general Lie triple

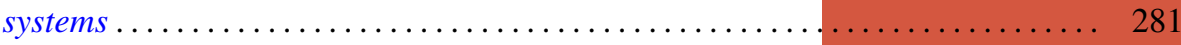

Laurent Siebenmann, A characterization of free projective planes . . . . . . . . . 293

Edward Silverman, Simple areas.................................. 299

James McLean Sloss, Chebyshev approximation to zero .................. 305

Robert S. Strichartz, Isometric isomorphisms of measure algebras . . . . . . . . . 315

Richard Joseph Turyn, Character sums and difference sets . . . . . . . . . . . . 319

L. E. Ward, Concerning Koch's theorem on the existence of arcs . . . . . . . . . . 347

Israel Zuckerman, A new measure of a partial differential field extension ......... 357 